\title{
THE THEORY OF FUNCTIONS.
}

Theory of Functions of a Complex Variable. By A. R. ForsYTH, Sc.D., F.R.S. Cambridge, University Press, 1893. 8vo, $\mathrm{xxii}+682 \mathrm{pp}$.

The year 1893 was marked by the appeararice of four important treatises on the theory of functions. The second volume of Picard's Traité d'Analyse* presents, as no other one book has ever yet done, the ideas that originated with Riemann and have been developed by him and his followers. 'The first volume of the new edition of Jordan's Cours d'Analyset begins, to be sure, with the elements of the infinitesimal calculus, but it is only after such a treatment of the foundation on which analysis rests, enriched by the methods and dominated by the spirit of what is commonly known as the modern theory of functions of a real variable, that Cauchy's theory of functions can be developed in a manner that will be adjudged rigorous by the standards of this age.

On the other hand, Harkness and Morley's treatise, $\$$ like the work before us, is not confined to any one of the three theories due respectively to Cauchy, to Weierstrass, and to Riemann; and these are the first works to appear on the theory of functions in which all three theories are treated. While they have a common object, namely, to introduce the student to the whole field of the theory of functions, the manner in which this plan is carried out is quite different. The former writers feel keenly the advances that have been made in recent times in accuracy and rigor in analysis. 'They know that this work has as yet found scarcely any place in the text-books, and that a knowledge of it cannot be assumed on the part of the student who has just finished his differential and integral calculus. They, therefore, devote some introductory chapters to it and emphasize it throughout the subsequent developments of their subject. Our author, on the other hand, gives little heed to such matters. He takes the ground that the student who is interested in these questions can find them treated well enough elsewhere; and he does not regard it as part of his task to point out to the student such difficulties and thus to foster in him a critical spirit, nor does he shape his proofs, as Picard has done so successfully, so as to avoid these difficulties, or, if that is not possible, to meet them squarely. This, from the author's point of view.

* Reviewed in the Burletrin, vol. 3, p. 39.

+ Reviewed in the Bulletis, vol. 3, p. 135

$\ddagger$ Reviewed in the Butsutsin, vol. 3, p. 155. 
But we cannot stop with pointing merely to the loose form in which theorems are often stated and proofs given; it only too often happeus that the ideas on which the proofs rest are lacking in rigor, or that important matters are overlooked.

It is thus easy to understand that Cauchy's theory appears simpler to the author than Weierstrass's. For the difficulties in the former are hidden in the elements of the older analysis, supposed known; while those of the latter centre in theorems regarding series, of which, though they are equally elementary-in some respects even more so-elementary arithmetical proofs are not generally known. We would not be understood as objecting to the author's use of Cauchy's theory. It seems to us an excellent feature of this treatise that the author has always aimed to use that method which is best adapted to the proof of the proposition in hand; and certainly Cauchy's methods, supposing them to have been once rigorously established, often lead more directly to the desired result than Weierstrass's. Riemann's theory of the algebraic functions and their integrals is set forth. But the possibility of basing the whole theory of functions on the logarithmic potential function, which is the leading idea in Riemann's theory (and which is worked out in detail, for example, in Picard's treatise), is not clearly brought out.

In connection with this treatment of the theory of functions, however, a further point deserves attention. While the student gets, by the alternation in the use of Cauchy's, of Weierstrass's, and of Riemann's methods, a broader view of the subject as a whole, he fails to get a due appreciation of the homogeneity of each method by itself. This difficulty could be met, in great measure, by brief sketches of the lines along which important results, obtained by use of one method, would be established by the other method, such sketches to be accompanied by full references regarding details. It may be said that this is a matter of interest only to mature students. But it is for such that the whole treatise will be of greatest value. It gives them on its pages a "consecutive account of what may fairly be deemed the principal branches of the whole subject" of the general theory of functions of a complex variable, and the full lists of bibliographical references guide them in making a systematic study of those special subjects on which they wish to inform themselves in detail. The service that the author has rendered to them in compiling these lists is great and will be deeply appreciated.

One important method we miss altogether. Transformations are nowhere treated, the linear transformation in its relation to automorphic functions alone excepted. 'Ihus, though considerable space is devoted to algebraic functions 
and their integrals, the rational transformations of one algebraic configuration (Gebilde) into another, together with the invariants of such transformation-the moduli (Moduln) of the Riemann's surface-and the transformations of the periods of the integrals, in particular, of the elliptic integrals, are only mentioned in passing.

An excellent feature of the book, and a feature distinctly English, is the frequent introduction of problems, illustrative of the principles treated, for the reader to solve. It is a mistake too frequently made, alike by teachers and students, to believe that one understands a theorem because one can prove it. 'The real test is whether one can apply it, and for this test examples are useful.

A glossary of technical terms, an index of authors quoted, and a general index-the latter covering over 18 pages-are appended.

Chapter I is entitled General Introduction. In $\& 1$, a brief statement is made regarding the relation of the algebra of ordinary complex quantities, $a+b i$, to other multiple algebras; but it is impossible to make this relation clear in half a page. The geometric representation of the complex variable by a point in the plane or on the sphere of stereographic projection is explained, but no reason is given for regarding the infinite region of the plane as a point, it being apparently sufficient that, in the projection on the sphere, one point of the latter remains unprovided for.-If $u, v$ be any functions whatever of $x, y$, then $u+v i$ will be a function of $z=x+y i$ in the sense that, when $z$ is given, $w$ is determined. In the case of the ordinary functions (rational functions, functions defined by convergent power series, etc.), $z$ being taken as the independent variable, $u$ and $v$ are restricted,-between their partial derivatives with regard to $x$ and $y$ there exist, for example, certain relations. The author regards this restriction as seated in the fact that the complex variable $z=$ $x+y i$ has been operated on as an "irresoluble entity," and, generalizing, regards a function of $z$ as generated by an operation on $z$ as a whole, not on $x, y$ separately. 'This idea is not a new one. It is, however, open to attack, for it is not clear that the above requirement sifts out from the general class of functions, $u+v i$, the special class that is desired, since the kind of operation, here supposed arithmetical, to be performed on $z$ has not been restricted. For example, the projection of the $z$ plane on the $w$ plane is a geometric operation that affects $z$ as a whole and that defines $w$ geometrically as a function of $z$; and it is conceivable that a convergent arithmetic process exist which, operating on $z$ as an "irresoluble entity," would yield the same function.-On p. 15 , the definition," a point in the plane, at which two or more 
branches of a multiform function assume the same value, is called a branch-point," is inaccurate. Thus $z=0$ is not a branch-point of the function $w$, where $w^{2}=z^{2}(1-z)$. An accurate definition is given on p. 162. The choice of the word uniform for single-valued does not seem fortunate; still less so, the rendering of ausserwesentlich singuläre Stelle by accidental singularity. The author always speaks of rational algebraic functions and rational integral algebraic functions; the reason for inserting the word algebraic in such cases is not clear.

In Chapter II, the integral of a complex function is defined, its existence being assumed, and elementary theorems regarding such integrals are established. On p. 20, the theorem is stated: "When a function is expressed in the form of a series, which converges uniformly and unconditionally, the integral of the function along any path of finite length is the sum of the integrals of the terms of the series along the same path, provided that path lies within the circle of convergence of the series." The requirement of unconditional convergence is irrelevant. In $\$ 1 \%$, Cauchy's fundamental theorem: "If a function $f^{\prime}(z)$ be holomorphic throughout any region of the $z$ plane, then the integral $f f(z) d z$, taken round the whole boundary of that region, is zero," is proved by double integration, no mention being made of other proofs. In the statement of the theorem of $\S 20$, the words "which is not a zero of $f(z)$ " should be omitted. One is at a loss to see why, in $\S 21$, so careful a proof is given that differentiation under the sign of integration is allowable. Usually, such matters are not touched upon. The chapter closes with some good examples in illustration of the preceding principles.

Chapter III begins with a careful proof of Cauchy's theorem regarding the expansion of a function into a power series. Laurent's theorem (p. 4\%) is stated as follows: "A function which is holomorphic in a part of the plane bounded by two concentric circles with centre $a$ and finite radii can be expanded in the form of a double series of integral powers, positive and negative, of $z-a$, the series converging uniformly and unconditionally in the part of the plane between the circles." 'The error here made regarding uniform convergence occurs repeatedly throughout the book. Consider, for example, the series

$$
1+z+z^{2}+\ldots
$$

Let $r$ be any positive real number less than 1 . Then this series converges uniformly within a circle of radius $r$ about the point $z=0 . \quad r$ can be taken as near to 1 as desired and the convergence will still be uniform. Nevertheless, the series 
does not converge uniformly within the unit circle about $z=0$, for the remainder after $n$ terms is

$$
\frac{z^{n}}{1-z}
$$

and, after a positive quantity $\epsilon$ has been chosen at pleasure, it is evidently not possible to find a positive integer $v$ such that, for all values of $n>v$, the absolute value of this remainder will lie below $\epsilon$, no matter how $z$ be now chosen within the unit circle about $z=0$. A power series always converges absolutely within its circle of convergence, but it may not converge uniformly. If it does, however, then it converges uniformly within and on this circle. In particular, a power series can converge absolutely for all points of the plane, but it cannot converge uniformly for this region, unless it be a constant. 'Thus, incorrect statements occur on pp. 76, 90, $13 \%, 142$, and elsewhere. In fact, the author often requires of a series whose terms are not even functions of a variable that it converge uniformly! Cf. pp. $76,83,85$. In the definition of uniform convergence, given on p. 12\%, the essential point is not stated, and we almost fear that it has escaped the author's attention.- $\$ \$ 32,33$ treat of the singularities of a single-valued function. It is tacitly assumed that these are isolated poles or essential singularities. In $\$ 33$, the behavior of a single-valued function in the neighborhood of an essential singularity is considered. The investigation is based on the theorem that, if a function $f(z)$ be holomorphic at all points in the neighborhood of $z=a$, then either (1) it is holomorphic at $z=a$ also ; or (2) there exists a series of values $z_{1}$, $z_{2}, \ldots, \lim z_{i}=a$ for $i=\infty$, such that $\lim f\left(z_{i}\right)=\infty$ for $i=\infty$. This theorem is not clearly established by the considerations of these paragraphs; it is, however, evident that the author has these relations clearly in mind. Then: "Further, a uniform function must be capable of assuming any value $C$ at an essential singularity." Now the function is not defined by the power series in the singular point, and whether we say, with Continental mathematicians, that it shall not be defined at all at this point or, with the author, that it shall be defined to have all values is, perhaps, a matter of taste. The important fact is this: let $C$ be any real or complex quantity, let a circle of arbitrary radius be drawn about the essential singularity, and let $\epsilon$ be an arbitrarily chosen positive quantity. Then it is always possible to find points within the circle (exclusive of the centre) in which the difference between $C$ and the value of the function is, in absolute value, less than $\epsilon$. The function may or may not attain the value $C$. 'Thus the function

$$
1+z+z^{2} / 2 !+\ldots
$$


never attains the value 0 . This fact is not brought out. Cf. also p. 64, Cors. I, II, and p. 219. The chapter ends with a clear account of Weierstrass's method of continuation of an analytic function and Schwarz's continuation by the principle of symmetry.

Chapter IV discusses single-valued functions, in particular, rational functions. Theorem I reads: "A function which is constant throughout any region of the plane not infinitesimal in area, or which is constant along any line not infinitesimal in length, is constant throughout its region of continuity"; and at the end of the proof: "It should be noted that, if in the first case the area be so infinitesimally small and in the second the line so infinitesimally short that consecutive points cannot be taken, then the values at $a$ of the derivatives cannot be proved to be zero, and the theorem cannot then be inferred." Here is the doctrine of infinitesimals so popular in earlier times. That doctrine regards an infinitesimal not as a variable, but as a constant, an "infinitely small constant," having geometric existence (e.g., an "element" of arc, area, etc.); and hence two fixed points on a line that do not coincide need not be situated a definite distance apart from each other. But this does not correspond to our intuition of a line, and geometric intuition was formerly the foundation on which arithmetic rested. In recent times, arithmetic has been developed independently of geometry, and it now stands on the firmest foundation known to mathematicians. In its turn, it has thrown light on the nature of our geometric axioms; and not the least important result that it has achieved is the demonstration of the impossibility of such a doctrine of infinitesimals as the one the author holds. Many other passages may be cited in this connection; of such the following are a few: p. 29, end; p. 45, near end; p. 201, middle; p. 22\%, Cor. II, note. On pp. 60, 61, we read: "Further, in any finite area of the region of continuity of a function that is subject to variation, there can be at most only a finite number of its zeros, when no point of the boundary of the area is infinitesimally near an essential singularity." The words "infinitesimally near" should be omitted; for, if the region contains an infinite number of zeros, there will be at least one essential singularity on the boundary. In the proof that follows, the inference that the zeros would fill out a continuous line or area is incorrect. Cf. also pp. 206, 218. In the subsequent pages of the chapter, the fundamental theorems relating to rational functions are established.

In Chapters V, VI, VII, the investigations of Weierstrass and Mittag-Leffler on single-valued transcendental functions, together with important examples given by Tannery and Poincaré, are treated at great length (pp. 74-148). In the 
last three lines of $\$ 50$ : "Further, $z=\infty$ is an essential singularity of the function; for it is an essential singularity of each of the factors on account of the exponential element in the factor," the reason assigned is wrong, as is shown by the following example:

$$
\stackrel{I}{I}_{0}^{\infty} p_{n}, \quad \text { where } p_{0}=e^{z}, \quad \eta_{n}=e^{\frac{1}{(n+1) !} z-\frac{1}{n !} z}, \quad n>0 .
$$

On p. $7 \%, 1.17$, "... and taking $\epsilon \ldots$," read $k$ for $\epsilon$. On p. 79, the author wishes to prove that an infinite product, $G(z)$, has an ordinary zero in a point in which one of its factors has an ordinary zero. For this purpose he says (I. 8): "Since the product $G(z)$ converges unconditionally, no product constructed from its factors $E$, say from all but one of them, can be infinite." The property in question does not follow from the unconditional convergence, nor even from the uniform convergence. It follows from the latter, combined with the fact that the individual factors are analytic functions throughout a common region.

In $\$ 82$, Weierstrass's theorem regarding the expression of a series of analytic functions $\Sigma f_{i}(z)$ as a series of positive and negative powers of $z$ is stated inaccurately, and the proof is confused. Weierstrass long ago published* his proof of this theorem, which is a model of clearness and rigor, and which the author would have done well to translate literally. The further theorem that such a series $\Sigma f_{i}(z)$ can be differentiated term by term is omitted. When such series arise, they are so differentiated without further ceremony (v. \$ 124). In $\$ 5 \%$, Weierstrass's $\sigma$-function is defined. The main results obtained in these chapters consist in the establishment of the various forms in which, as Weierstrass and Mittag-Leffler have shown, single-valued functions, especially those with a single essential singularity, can be expressed when their zeros and poles are known. Good examples are given. The investigations of Cantor on clusters of points find no place in the book. Such clusters cannot be avoided in Mittag-Leffler's theorems, so the author skilfully restricts his treatment to the case where the derived cluster is the circumference of a circle (p. 11\%). In $\S 8 \%$, Poincaré's examples of functions with lacunary spaces are considered, but the reasoning that the author gives is wrong. It does not follow from the fact that the terms of an infinite series

$$
\sum_{i}^{\infty} \frac{A_{i}}{z-b_{i}}
$$

* Monatsher. d. Berl. Akad., 1880. 
have poles clustering about a point $b$, that this point will be an essential singularity for the function defined by the series, as is here assumed. Consider, for example, the series

$$
-\frac{1}{z}+\frac{z}{z^{2}+1}+\sum_{1}^{\infty}\left(\frac{z}{z^{2}+\frac{1}{n+1}}-\frac{z}{z^{2}+\frac{1}{n}}\right)
$$

which has the value 0 for all values of $z$ for which its terms are defined, and which can be thrown at once into the above form. Its terms have poles clustering about the point $z=0$, but this is not a singularity of the function. The restriction that Poincaré makes on the coefficients $A$ is essential. Cf. Poincaré's memoir in the Amer. Jour. of Math., vol. 14.The results obtained in the memoirs under consideration are, indeed, given in these chapters, but the careful proofs of the original are not preserved.

With Chapter VIII, the treatment of multiple-valued functions begins. This chapter is more a description than a careful treatment of the general properties of such functions. Branchpoints are surrounded with loops, and the Riemann's surface is not introduced till later. The transformations that lead to the separation of the branches of an algebraic function at a singular (multiple) point are considered, but no proof is given that a finite number of applications of such transformations will eventually effect the desired separation; indeed, no proof is given that an algebraic function is analytic. Surely we could have spared some of the 74 pages of Chapters V-VII better than to do without these theorems. - The case treated by Clebsch and Gordan in their Abelsche Functionen of a curve with only double points and cusps (the corresponding Riemann's surface having, accordingly, only simple branch-points) is designated by the author (\$98) as "of considerable importance, though limited in character." From the important point of view that two algebraic configurations (Gebilde) are equivalent when one can be transformed rationally into the other, there is here no limitation whatever.

Chapters IX-XIII are devoted to periodic functions. Integrals with moduli of periodicity are discussed, and Hermite's method is described. Functions defined by definite integrals are considered, and the double-circuit integrals introduced by Jordan and Pochhammer are referred to, but not discussed. Jacobi's arithmetic proof that a single-valued function cannot have more than two periods is given, but no geometric proof, like that given, for example, in Briot and Bouquet's Fonctions elliptiques, $2 \mathrm{~d}$ ed., bk. IV, ch. III, is mentioned. The treatment of simply periodic and doubly periodic functions, in Chapter $X$, would have been rendered clearer if it had been illus- 
trated by the corresponding conformal representations. The transformations $Z=e^{\frac{2 \pi i}{\omega} z}$ and $Z=\tan \frac{\pi z}{\omega}$ are studied arithmetically, and these functions are used as prime functions in terms of which simply periodic functions are expressed. The theorem near the top of p. 215 is not stated accurately. The theorem of $\$ 114$ is incorrect; consider $e^{z^{2}} \tan z$. The theorem that no rational integral function can be simply periodic is omitted, though the corresponding theorem for double periodicity is stated and proved. Next, the fundamental theorems regarding doubly periodic functions are developed, and the general differential equations of Jacobi's and of Weierstrass's elliptic functions are deduced analytically from these theorems. -Doubly-Periodic Functions of the Second Order is the title of Chapter XI. Weierstrass's $\sigma_{-}, \zeta_{-}$, and $\wp$-functions are introduced. Throughout these chapters, the general line of thought is presented clearly, but details are often omitted which the student cannot supply, as, for example, in $\S 124$, already referred to.-Chapter XII is on Pseudo-Periodic Functions, of which $\theta$ and $\sigma$ are examples, and Hermite's application to the solution of Lamés equation ( $\$ 140)$ is discussed.-In Chapter XIII, Weierstrass's theorem relating to analytic functions with an algebraic addition-theorem is taken up. Phragmén's proof is referred to, but a different proof is given by the author. This latter proof is, however, incomplete in some essential points. The assumption that forms the starting-point in Weierstrass's theorem is that, between three convergent power series, $P_{1}\left(u-u_{0}\right), P_{2}\left(v-v_{0}\right), P_{3}\left(w-\overline{u_{0}+v_{0}}\right)$, elements of the same analytic function, $\phi(u)$, there exist, when $w$ is put equal to $u+v$, an algebraic relation,

$$
G\left\{P_{1}\left(u-u_{0}\right), P_{2}\left(v-v_{0}\right), P_{3}\left(u+v-\overline{u_{0}+v_{0}}\right)\right\} \equiv 0 ;
$$

and the existence of the same algebraic relation between $\phi(u)$, $\phi(v), \phi(u+v)$ must first be established. All this, the author omits. He further assumes that if $\phi(u)$ is defined for $u$ and for $v$, then it is also defined for $u+v$. But this is not true, even in general, of all analytic functions. When these results have been established, however, the further proof along the lines that Phragmén follows is much simpler than the one the author gives.

In Chapter XIV, entitled Connection of Surfaces, the author gives a clear and simple account of this subject. Only bifacial surfaces are considered. Systems of irreducible circuits are treated, and the way is prepared for Chapter XV, Riemann's Surfaces. The first pages of this chapter are, however, far from clear. Thus the author speaks ( $\$ 173)$ of "the theorem that 
sheets of a Riemann's surface are joined along lines"; and the form of the remainder of the paragraph is that of a proof of this "theorem". In fact, in the next two paragraphs as well, it is difficult to distinguish between what is intended as definition and what is intended as proof. Cor. I on p. 341 is not correct; consider $w^{2}=1-z^{4}$ and draw the branch-lines as the straight lines $-1,0,+1$, and $-i, 0,+i$. A careful study of some simple examples of conformal representation, illustrated by skilfully designed diagrams, might well precede such general discussion. Examples are, indeed, given in $\S 176$, but they are inadequate. From $\S 178$ on, the presentation is much clearer. The relation between the connectivity $(2 p+1)$, the number of simple branch-points, and the number of sheets is established and the existence of canonical systems of irreducible circuits is made clear; the question of how such a system is to be found in a given case is not considered. Finally, the case of a Riemann's surface with only simple branch-points-a generalization, in a way, of the hyperelliptic surface of two sheets-is discussed, and a canonical set of branch-lines is introduced, $\$ 189$. A simple figure would have made the result much more striking. The way in which a canonical system of irreducible circuits is to be taken on such a surface is at once evident.

Chapter XVI, Algebraic Functions and their Integrals, is a brief sketch of some of the most important relations. Here, again, it is to be regretted that the author makes no use of conformal representation, especially as he could thus give some important illustrations of the subjects of the previous two chapters. We mention, in passing, that sufficient restrictions have not been made to justify the conclusion at the end of $\S 193$; the equation $F\left(w^{\prime}, z\right)=0$ may be reducible. The integral of an algebraic function on a Riemann's surface with simple branch-points is considered. The chapter closes with the definition of the Abelian integrals of the three kinds.

'The further treatment of algebraic functions is now interrupted by the proof of the existence theorem, Chapter XVII, in which Schwarz's proof is given. The problem to be discussed is stated clearly in the opening paragraphs of the chapter, but the reader who seeks here a rigorous exposition of Schwarz's rigorous analysis will seek in vain; for, as has already been pointed out, rigor of form nowhere marks the author's work and here such deficiency is particularly hampering. In the proof of any complicated theorem in limits, it is of prime importance that it be stated distinctly what magnitudes are chosen arbitrarily and what ones are chosen subsequently to and in a measure dependent on these. This obvious requirement the author does not observe. See, for example, $\$ 219$ in this chapter and $\S 8 \%$. Again, the line re- 
ferred to in $\mathrm{V}$ on p. 422 must not be tangent to the boundary-an essential condition that is not mentioned. But such criticisms as this might be made throughout the book.-The proof given in the second paragraph on p. 416 is not sound. 'The lemma on p. 410 is a truism as it stands; it should be amended to read: ". . and if $u_{1}$ and $u_{2}$ be the same for any two-dimensional part (no matter how small) of the common portion $T, \ldots$ At the close of $\S 227$ the boundary, it is stated, can be made a point. This statement is interesting in connection with Schwarz's words on the last page of the Auszug aus einem Briefe an Herrn F. Klein, Math. Ann., vol. 21, pp. 15\%-160; Ges. Werke, II., p. 305. In this letter, Schwarz indicates briefly how his method can be applied to closed surfaces. A fuller statement of the details would have been acceptable to the reader.

In Chapter XVIII, the study of Abelian integrals is again taken up. Relations between their periods are established and normal integrals are introduced. In $\$ 239$, Abelian functions are defined. The reason that the author gives for not defining an Abelian function as the inverse of an Abelian integral is extraordinary. He has shown in $\S 110$ that, if $z_{0}$ is any point within the region of continuity of an Abelian integral $w(z)$ and $A$ an arbitrarily assigned real or complex quantity, a path can be so chosen for the integral that $A-w\left(z_{0}\right)$ will be, in absolute value, as small as is desired. (He thinks he has shown that $A-w\left(z_{0}\right)$ can be made to vanish.) Because of these relations he says that $z$ can no longer be considered as a function of $w$ ! 'That is to say, before we can regard Cauchy's or Weierstrass's or Riemann's definition of a function as really defining a function, we must first make sure that such relations as the above do not exist! The real reason for the usual definition of the Abelian functions is, of course, in order to bring the inverse of the integral into the closest possible relation with single-valued functions. Now the inverse function is the root of an algebraic equation whose coefficients are single-valued functions, not, to be sure, of a single integral, but of $p$ integrals. These coefficients are defined as Abelian functions.-In closing, the author gives a brief sketch of the allied functions, the integrands of the Abelian integrals. We believe that the author is right in adopting Riemann's methods for the treatment of the algebraic functions and their integrals. For these methods have now been rigorously established, while in the algebraic treatment, which Noether has done so much toward developing, many details still remain to be worked out. But his object is not solely to lay the foundation of a systematic treatment; it is also, in part, to make the student acquainted with some of the simplest properties of these and of their allied functions. 
For this purpose, plane curves, rational transformations, and conformal representations are most useful means, and we regret that they have not appealed to the author.-An account of Appell's "factorial functions" and a discussion of the conditions under which the algebraic differential equation $f\left(\frac{d w}{d z}, w\right)=0$ defines $w$ as a single-valued function of $z$ conclude the chapter.

Chapter XIX, Conformal Representation: Introduction, opens with a discussion of the general problem of the conformal transformation of one surface on another, and several examples are given in illustration. With $\$ 25 \%$, the more special problem of the conformal transformation of one plane on another is taken up and a large number of examples, including some of the regular bodies, follow. The sequence of these examples might be amended with advantage, and the whole presentation would be clearer if better jllustrated by diagrams. The last twelve pages of the chapter are given to the linear transformation. Its fundamental properties are, indeed, set forth in clear analytic form; but surely the reader may demand that, on a transformation of such importance, the strongest possible geometric light be thrown.

Chapter XX begins with an account of Schwarz's investigations on Riemann's theorem regarding the conformal transformation of a singly connected surface on a circle. Schwarz gave a proof of the theorem under certain very general restrictions. Any account of Schwarz's work that is to be of real value to the student must state accurately the leading ideas on which the successive theorems rest, the chain of necessary conditions that determine the function completely on the supposition that it exist, and finally the argument by which the existence of such a function is established. For, the object of Schwarz's investigations is not to give a heuristic method, but an analytic proof. Now it may be that the author intended a presentation of this sort; but if he did, he has certainly been unfortunate in his style. The conformal transformation on a circle of polygons whose sides are straight lines or arcs of circles is treated and examples are given. In $\$ 269$, we read: "For general values of $\alpha, \beta, \gamma$ the integral function $w$ is an Abelian transcendent..." This is true only when $\alpha, \beta, \gamma$ are rational numbers. Next comes a study of the case that the polygon in the $w$ plane is a triangle whose sides are arcs of circles. The corresponding differential equation of the third order and its resolvent linear differential equation of the second order are obtained. Figures are given for the regular bodies and these diagrams illustrate remarkably well the divisions of the sphere. 'The equations of the regular bodies are deduced directly in the non-homo- 
geneous form. The chapter closes with a proof of Schwarz's theorem that " the only equations $\chi(z, w)=0$ which can give families of algebraical curves in the $z$ plane as the conformal equivalent of the parallel lines, $u=$ constant, in the $w$ plane, are such that $z$ is connected by an algebraical equation either with $w$, or with a simply periodic function of $w$, or with a doubly periodic function of $w$." (p. 579.)

'The last two chapters of the treatise are entitled, respectively, Groups of Linear Substitutions, and Automorphic Functions. The first of these takes up rather fully the general properties of such groups, without going into the more subtle questions that arise in connection with them, and it forms a good introduction to the study of these groups. In the last chapter, automorphic functions are defined and illustrated by the simplest examples of such functions, the rational functions belonging to the regular bodies and the elliptic modular functions. Here, it would have been easy for the author to point out the essential distinction between the elliptic functions of Jacobi and Legendre and those of Weierstrass in relation to their invariance as regards the substitutions of the modular group. Poincaré's pseudo-automorphic $\Theta$ series are introduced and some properties of automorphic functions, defined as the quotient of such series, are deduced.

It will be seen from the points to which attention has been called that the book is not one that can safely be put into the hands of the immature student for a first introduction to the study of the theory of functions. But the student who is already familiar with its elements and who has acquired some degree of critical power will find its pages incentive to valuable work in this wide field.

Harvard University, February, 1895.

W. F. OsGood. 